\title{
Tangshen formula improves inflammation in renal tissue of diabetic nephropathy through SIRT1/NF-кB pathway
}

\author{
YUE-GUANG DU ${ }^{1}$, KE-NA ZHANG ${ }^{1}$, ZONG-LEI GAO $^{2}$, FENGJIAO DAI ${ }^{2}$, XI-XI WU ${ }^{2}$ and KE-FU CHAI ${ }^{2}$ \\ ${ }^{1}$ Department of Pathology; ${ }^{2}$ National Traditional Chinese Medicine Clinical Research Center, \\ Zhejiang Chinese Medical University, Hangzhou, Zhejiang 310053, P.R. China
}

Received January 20, 2016; Accepted February 24, 2017

DOI: $10.3892 /$ etm.2017.5621

\begin{abstract}
The present study investigated the mechanism underlying the anti-inflammatory effects of Tangshen formula (TS) in Sprague Dawley (SD) rats with diabetic nephropathy (DN). A rat model of DN was established by intraperitoneal injection of $1 \%(40 \mathrm{mg} / \mathrm{kg})$ streptozotocin and administration of a high fat and glucose diet. Subsequently, SD rats were randomly divided into six groups $(\mathrm{n}=8)$ : A DN group, a valsartan group, a high-dose TS group, a middle-dose TS group, a low-dose TS group and a control group with normal SD rats. Once rats received their allocated treatment for 12 weeks, body weight and kidney weight were recorded, and fasting blood glucose, ratio of urinary protein, $\beta_{2}-\mathrm{MG}$ and creatinine clearance rate were determined. Furthermore, hemodynamic indices, including plasma viscosity and whole blood reduction viscosity were detected. Immunohistochemistry was used to detect the infiltration of macrophages in the kidneys of rats. Reverse transcription-quantitative polymerase chain reaction and western blotting were performed to investigate the activation; mRNA and protein expression levels of monocyte chemoattractant protein-1 (MCP-1), macrophage migration inhibitory factor (MIF), nuclear factor- $\kappa \mathrm{B}(\mathrm{NF}-\kappa \mathrm{B})$ and sirtuin-1 (SIRT1) in each group. In comparison with the DN group, each biochemical indicator of rats in the high-dose TS group was significantly decreased $(\mathrm{P}<0.05)$. Blood viscosity in each treatment group was significantly decreased when compared with the DN group $(\mathrm{P}<0.01)$. Hematoxylin and eosin staining indicated that the infiltration of macrophages was significantly
\end{abstract}

Correspondence to: Professor Ke-Fu Chai, National Traditional Chinese Medicine Clinical Research Center, Zhejiang Chinese Medical University, 548 Binwen Road, Hangzhou, Zhejiang 310053, P.R. China

E-mail: ckf666@163.com

Abbreviations: DN, diabetic nephropathy; DM, diabetic mellitus; PKC, protein kinase C; FBG, fasting blood glucose; UAER, urinary albumin excretion rate; $\mathrm{Ccr}$, creatinine clearance rate

Key words: diabetic nephropathy, Tangshen formula, macrophages, nuclear factor- $\kappa \mathrm{B}$, sirtuin decreased in the high-dose TS group when compared with the DN group $(\mathrm{P}<0.01)$. mRNA and protein expression levels of MCP-1 and MIF in the high-dose TS group were significantly decreased when compared with the DN group $(\mathrm{P}<0.05)$. In the treatment groups, SITR1 mRNA expression levels were significantly increased, whereas the mRNA expression levels of $\mathrm{NF}-\kappa \mathrm{B}$ were significantly decreased $(\mathrm{P}<0.01)$. Western blotting results indicated a significant decrease in the protein expression levels of acetylated NF- $\kappa \mathrm{B}$ in the treatment groups when compared with the DN group $(\mathrm{P}<0.01)$ and the propensity of protein expression of the other inflammatory factors were consistent with the mRNA findings. The results of the high-dose TS group were similar to those of the valsartan group. The present study indicates that TS was able to activate SITR1, which lead to NF- $\kappa \mathrm{B}$ deacetylation, thus reducing the release of inflammatory factors and decreasing the severity of diabetic nephropathy.

\section{Introduction}

Diabetic nephropathy (DN) is the most common and serious complication of diabetes (1). China has around 400 million diabetic patients and $20-30 \%$ of these progress to DN, and $20-40 \%$ of patients with DN develop end-stage renal disease, which is severely harmful to human health (2). Western researchers have indicated that the pathogenesis of $\mathrm{DN}$ and glucose metabolism are predominantly caused by non-enzymatic glycation, polyol pathway activation, protein kinase $\mathrm{C}$ activation, dyslipidemia, hypertension, renal hemodynamic changes, oxidative stress, vasoactive substances, cytokines and several other genetic factors (3). However, researchers of traditional Chinese medicine believe that diabetic nephropathy belongs to the category of diabetes disease (4). In traditional Chinese medicine, it is believed that the pathogenesis of dryness and heat is due to a deficiency of Yin. Yin deficiency is the 'root' cause of the disease, while dryness and heat are the symptoms (5). A prolonged state of Yin deficiency may result in deficiency of both Qi and Yin, as well as a deficiency of Yin and Yang (4). This phenomenon may also cause incidental symptoms, including blood stasis, phlegmatic hygrosis and turbidity toxin. Blood stasis or blood stagnation is an important underlying pathology of many diseases according to Traditional Chinese Medicine. It is described as a slowing or pooling of the blood due to disruption of the heart and is often 
understood in biomedical terms as hematological disorders, including hemorrhage, congestion, thrombosis and local ischemia (microclots) or tissue changes (6). Phlegmatic dampness is created by impaired digestion and is primarily located in the lungs and large intestine (7). When the dampness is stored in the large intestine, it may lead to mucus-lined, loose or sticky stools that are difficult to clean up after or diarrhea with undigested bits of food (8). The Essential Pathogenesis of Diabetes Mellitus in traditional Chinese medicine refers to turbidity toxin as excess nutrition and the absorption of incompletely digested food, including protein and fat (9). It accumulates gradually to form a sticky sputum and ultimately the aggregated particles form a large block. This may lead to inflammation and suppuration, and may affect the transport and metabolism of nutrients (10). This mechanism is somewhat similar to the formation of atherosclerosis in Western medicine (4). Western medical practices have adopted various methods for controlling high blood glucose, blood pressure, low-protein diet, correcting lipid metabolism disorders and alternative symptomatic treatment for treating DN; however, none of these approaches are completely effective in preventing the progression of DN $(11,12)$. The current study of traditional Chinese medicine for the treatment and prevention of $\mathrm{DN}$ is primarily based on compound prescriptions and treatment is performed using Tangshen capsules and Tangshenkang (13), which, in traditional Chinese medicine are believed to be beneficial in supplementing Qi, nourishing Yin, promoting circulation and removing stasis. Tong Luo Yi Shen Zi Yin prescription is used as an alternative treatment that has a key purpose in promoting blood circulation to remove obstructions in the blood vessels and to improve the blood supply to the kidneys (14). Chinese medicinal treatment applies multi-channel and multi-target activities to achieve positive and effective therapeutic effects, thus the application of traditional Chinese medicine has great potential in the prevention of DN.

According to the traditional Chinese medicine theory of Professor Kefu Chai, it is believed that the basic pathogenesis of early- and medium-stage DN is the deficiency of both Qi and Yin, which results in blood stasis (15). Previous results have indicated that a Chinese herbal compound prescription, Tangshen (TS) formula, has demonstrated good clinical efficacy in managing early-stage DN, as it is believed in traditional Chinese medicine that it supplements Qi, nourishes Yin, improves blood flow and removes obstructions in the blood vessels (16). In the present study, the molecular mechanisms of TS formula in a DN rat model were analyzed, providing a deeper insight into the effects of TS formula in the treatment of DN.

\section{Materials and methods}

Animals. A total of 50 specific pathogen-free male Sprague Dawley rats (age, 6-8 weeks; body weight, 160-200 g) were purchased from Shanghai Sippr-BK Laboratory Animal Co., Ltd. [Shanghai, China; (certificate of approval, SCXK (Shanghai); 2008-0016]. Rats were bred in the Research Center of Zhejiang Chinese Medical University (Zhejiang, China), with the experimental license number of SYXK 2013-0184 (clean grade). Rats were maintained in the following experimental conditions: A 12-h light-dark cycle; a maintained temperature of $18-22^{\circ} \mathrm{C}$ with a relative humidity of $40-70 \%$; and access to food and water ad libitum. All animal experiments were approved by Zhejiang Chinese Medical University Animal Ethics Committee (Zhejiang, China).

Pharmacological agents. TS formula for one human body daily dosage is constituted of six different Chinese herbs, including Radix puerariae (15 g), astragalus (18 g), Ligustrum lucidum (15 g), Lucid ganoderma (15 g), Salvia miltiorrhiza (9 g) and Rheum rhabarbarum (6 g), which were all purchased from the Pharmacy Department of Zhejiang Provincial Hospital of Traditional Chinese Medicine (Zhejiang, China). Once herbs were combined, water $(\mathrm{v} / \mathrm{w})$ at eight times of the herbs weight was added. Subsequently, the medicinal herbs were decocted on a slow fire for $1 \mathrm{~h}$ and the remaining fluid was collected. The remaining pharmacological agents were continuously processed by adding water ( $\mathrm{v} / \mathrm{w})$ six times for a further $1 \mathrm{~h}$ on the slow fire. The two remaining fluids were merged and the compounds were condensed at a final concentration of $2 \mathrm{~g} / \mathrm{ml}$ and stored at $4^{\circ} \mathrm{C}$. The positive agent, valsartan, was purchased from Beijing Pharma Ltd. (Beijing, China). Valsartan was dissolved in sodium carboxymethyl cellulose.

Reagents and instruments. Streptozotocin (STZ) was purchased from Sigma-Aldrich (Merck Millipore, Darmstadt, Germany) and the reverse transcription-quantitative polymerase chain reaction (RT-qPCR) iTaq ${ }^{\mathrm{TM}}$ Universal SYBR ${ }^{\circledR}$ Green One-Step kit was purchased from Bio-Rad Laboratories, Inc.(Hercules, CA, USA). The primary antibody of monocyte chemoattractant protein-1 (MCP-1; sc-1785; 1:800), macrophage migration inhibitory factor (MIF; sc-20,121; 1:800), nuclear factor- $\kappa \mathrm{B}$ (NF-кB; sc-372; 1:500), sirtuin-1 (SIRT-1; sc-15,404; 1:300) and $\beta$-actin were all purchased from Santa Cruz Biotechnology, Inc. (Dallas, TX, USA). The IgG-horse radish peroxidase (1:3,000; cat no. M209180) was obtained from Pierce (Thermo Fisher Scientific, Inc., Waltham, MA, USA). Hemorheology was performed using a 7020-automatic biochemical analyzer (Hitachi, Ltd., Tokyo, Japan). Western blotting and PCR were performed using a T Personal thermocycler (Biometra $\mathrm{GmhH}$, Göttingen, Germany) and Mini-PROTEAN electrophoresis system (Bio-Rad Laboratories, Inc., Hercules, CA, USA).

Establishment of DN model and treatment. Prior to experiments, a total of $50 \mathrm{SD}$ rats were fed for 1 week for adaptation and urine protein and blood glucose levels were detected. A total of 3 rats exhibited abnormal results and were subsequently sacrificed. The remaining $47 \mathrm{SD}$ rats were intraperitoneally injected with $1 \% \mathrm{STZ}$ at $40 \mathrm{mg} / \mathrm{kg}$ and fed high-fat glucose fodder, containing 10\% egg yolk powder, $10 \%$ lard, $10 \%$ sucrose, $0.5 \%$ cholesterol and basic feed. This fodder was processed and disinfected by the Zhejiang Academy of Medical Sciences (Zhejiang, China). Another 8 rats were injected with an equal volume of vehicle $(0.1 \mathrm{M}$ citrate buffer; $\mathrm{pH} 4.2$ ) to represent the normal control. A total of $72 \mathrm{~h}$ after the injection, tail vein blood was collected and serum glucose was detected in all rats. The diabetic rat model was successfully established with serum glucose $\geq 16.7 \mathrm{mmol} / 1$. Diabetic rats were randomly divided into groups: Diabetic nephropathy group (DN group; $n=8$ ), valsartan group (valsartan group; $\mathrm{n}=8$ ), and low-, medium- and high-dosage of TS formula 
groups (all $n=8)$. Rats injected with vehicle were considered as the normal control group $(n=8)$. Rats of the valsartan group were treated with valsartan solution $(25 \mathrm{mg} / \mathrm{kg} /$ day $)$ and the rats in the TS formula groups were treated with TS formula, $6.5 \mathrm{~g} / \mathrm{kg} /$ day (low dose), $13 \mathrm{~g} / \mathrm{kg} /$ day (medium dose) and $26 \mathrm{~g} / \mathrm{kg} /$ day (high dose), respectively. The rats in these groups were observed for 12 weeks; 12 weeks following treatment, all rats were sacrificed and the renal cortex was collected for pathological observation and further investigation.

Specimen collection. Following treatment, 24-h urine collection was performed. Under empty stomach conditions, 3\% pentobarbital sodium (Sigma-Aldrich; Merck Millipore; cat no. P376; $45 \mathrm{mg} / \mathrm{kg}$ ) anesthesia was administered to acquire serum, plasma and whole blood samples. Rat kidneys were weighed and either stored in $10 \%$ formalin solution for pathological detection or stored at $-80^{\circ} \mathrm{C}$ for protein and mRNA detection.

Blood and urine biochemical index determination. The 24-h urine collected was centrifuged at 1,200 g/min for $10 \mathrm{~min}$ at $4^{\circ} \mathrm{C}$ and the supernatant was obtained. The automatic biochemical analyzer tested the blood for glucose, blood urine creatinine and serum creatinine levels and the endogenous creatinine clearance rate was calculated. The micro albuminuria and $\beta 2$-microglobulin ( $\beta 2-\mathrm{MG}$ ) levels were detected using the Rat $\beta 2$-microglobulin, BMG/ $\beta 2-\mathrm{MG}$ (Sangon Biotech Co., Ltd., Shanghai, China; cat no. D730498) and micro albuminuria ELISA kits (Wuhan Elabscience Biotechnology Co., Ltd., Wuhan, China; cat no. E-EL-R0025). Hemorheology indices, including blood viscosity and whole reduced blood viscosity, were determined using an automatic blood rheometer.

Hematoxylin and eosin $(H \& E)$ and periodic Schiff reaction (PAS) to observe pathological changes. Kidney tissues were fixed in $10 \%$ neutral formalin at $4^{\circ} \mathrm{C}$ overnight for dehydration. Tissues were heated at $120^{\circ} \mathrm{C}$ for $10 \mathrm{~min}$ in an autoclave for antigen retrieval and then dehydrated in an ascending grade of ethanol, washed with xylene and embedded in paraffin. The paraffin-embedded samples were cut into sections $3 \mu \mathrm{m}$ thick for histopathological examination. Tissue sections were de-paraffinized in xylene and stained with H\&E for $20 \mathrm{~min}$ at room temperature. For PAS staining, paraffin slides were de-paraffinized, rehydrated and washed in water. Slides were placed in $2 \%$ Periodic Acid for $15 \mathrm{~min}$ and stained with Schiff's reagent for $25 \mathrm{~min}$ at room temperature. Counter-staining with filtered hematoxylin was then conducted. Following washing three times, slides were rapidly dipped in acid alcohol twice and slowly dipped in ammonia four times. Slides were then dehydrated and cover slipped. The glomerular, renal tubular structure and matrix hyperplasia were studied under a light microscope using H\&E and PAS staining.

Infiltrating macrophages in renal tissues. EnVision two-step method was used for immunohistochemical staining. Following antigen retrieval, the sections were incubated with cluster of differentiation (CD) 68 primary antibody (1:50) at $4^{\circ} \mathrm{C}$ overnight. Samples were washed with PBS and incubated with EnVision working solution for $30 \mathrm{~min}$. DAB and hematin
Table I. Changes in weight, kidney weight and kidney hypertrophy index following treatment.

\begin{tabular}{lcccc}
\hline Groups & $\mathrm{N}$ & Weight $(\mathrm{g})$ & $\begin{array}{c}\text { Kidney } \\
\text { weight }(\mathrm{g})\end{array}$ & $\begin{array}{c}\text { Kidney } \\
\text { hypertrophy } \\
\text { index }\left(\mathrm{x} 10^{-3}\right)\end{array}$ \\
\hline Control & 8 & $541.25 \pm 23.81$ & $3.19 \pm 0.15$ & $5.94 \pm 0.71$ \\
DN & 8 & $291.62 \pm 14.58^{\mathrm{a}}$ & $3.85 \pm 0.07^{\mathrm{a}}$ & $13.24 \pm 0.82^{\mathrm{a}}$ \\
TS $^{\text {Low }}$ & 8 & $335.28 \pm 18.22$ & $3.66 \pm 0.11^{\mathrm{b}}$ & $10.93 \pm 0.67^{\mathrm{b}}$ \\
TS $^{\text {Med }}$ & 8 & $346.63 \pm 10.64$ & $3.50 \pm 0.09^{\mathrm{b}}$ & $10.10 \pm 0.35^{\mathrm{b}}$ \\
TS $^{\text {Hi }}$ & 8 & $362.25 \pm 22.68^{\mathrm{b}}$ & $3.49 \pm 0.15^{\mathrm{b}}$ & $9.66 \pm 0.3^{\mathrm{b}}$ \\
Valsartan $^{\mathrm{b}}$ & 8 & $355.25 \pm 17.94^{\mathrm{b}}$ & $3.48 \pm 0.07^{\mathrm{b}}$ & $9.81 \pm 0.58^{\mathrm{b}}$ \\
\hline
\end{tabular}

${ }^{\mathrm{a}} \mathrm{P}<0.05$ vs. control group; ${ }^{\mathrm{b}} \mathrm{P}<0.05$ vs. $\mathrm{DN}$ group. Data are presented as the mean \pm standard error of the mean. TS, Tangshen; $\mathrm{TS}^{\text {Low }}$, low dose $(6.5 \mathrm{~g} / \mathrm{kg} /$ day $)$ of Tangshen formula; TS $^{\text {Med }}$, medium dose (13 g/kg/day) of Tangshen formula; $\mathrm{TS}^{\mathrm{Hi}}$, high dose of Tangshen formula (26 g/kg/day) of Tangshen formula; Valsartan, $25 \mathrm{mg} / \mathrm{kg} / \mathrm{day}$ of valsartan; DN, diabetic nephropathy.

Table II. Changes in biochemical criterion following treatment $(\mathrm{n}=8)$.

\begin{tabular}{lcccc}
\hline Group & $\begin{array}{c}\text { FBG } \\
(\mathrm{mmol} / \mathrm{l})\end{array}$ & $\begin{array}{c}\text { UAER } \\
(\mu \mathrm{g} / \mathrm{min})\end{array}$ & $\begin{array}{c}\beta_{2}-\mathrm{MG} \\
(\mathrm{ng} / \mathrm{ml})\end{array}$ & CCR \\
\hline Control & $9.25 \pm 0.98$ & $0.28 \pm 0.09$ & $45.02 \pm 2.79$ & $121.25 \pm 6.57$ \\
$\mathrm{DN}$ & $30.08 \pm 2.89^{\mathrm{a}}$ & $2.83 \pm 0.70^{\mathrm{b}}$ & $65.8 \pm 3.39^{\mathrm{a}}$ & $69.79 \pm 3.03^{\mathrm{b}}$ \\
$\mathrm{TS}^{\mathrm{Low}}$ & $28.96 \pm 2.61$ & $2.39 \pm 0.31$ & $59.15 \pm 3.6$ & $80.00 \pm 2.09$ \\
$\mathrm{TS}^{\text {Med }}$ & $29.52 \pm 2.93$ & $2.19 \pm 0.25^{\mathrm{c}}$ & $57.57 \pm 3.64$ & $84.80 \pm 3.48$ \\
$\mathrm{TS}^{\mathrm{Hi}}$ & $24.63 \pm 2.78^{\mathrm{d}}$ & $1.79 \pm 0.33^{\mathrm{c}}$ & $55.19 \pm 3.28^{\mathrm{c}}$ & $88.52 \pm 3.55^{\mathrm{c}}$ \\
Valsartan & $25.56 \pm 2.18^{\mathrm{d}}$ & $1.99 \pm 0.33^{\mathrm{c}}$ & $56.15 \pm 5.03$ & $90.76 \pm 5.42^{\mathrm{c}}$
\end{tabular}

${ }^{\mathrm{a}} \mathrm{P}<0.01$ and ${ }^{\mathrm{b}} \mathrm{P}<0.05$ vs. control group; ${ }^{\mathrm{c}} \mathrm{P}<0.05$ and ${ }^{\mathrm{d}} \mathrm{P}<0.01$ vs. $\mathrm{DN}$ group. Data are presented as the mean \pm standard error of the mean. TS, Tangshen; TS ${ }^{\text {Low }}$, low dose $(6.5 \mathrm{~g} / \mathrm{kg} /$ day $)$ of Tangshen formula; $\mathrm{TS}^{\mathrm{Med}}$, medium dose (13 g/kg/day) of Tangshen formula; $\mathrm{TS}^{\mathrm{Hi}}$, high dose of Tangshen formula (26 g/kg/day) of Tangshen formula; Valsartan, $25 \mathrm{mg} / \mathrm{kg} /$ day of valsartan; DN, diabetic nephropathy; FBG, fasting blood glucose; UAER, urinary albumin excretion rate; $\mathrm{CCR}$, creatinine clearance rate.

counterstaining were performed. Images were captured from five random fields under a light microscope (magnification, x400).

$R T$ - $q$ PCRfor detecting $m R N A$ expression levels. Total RNA was extracted from rat kidney tissue using TRIzol total RNA isolation reagent (Gibco; Thermo Fisher Scientific, Inc.) according to the manufacturer's protocol. Total RNA was reverse transcribed using the RT-PCR kit followed by qPCR detection. Primer sequences were as follows: SIRT1 (306 bp), forward 5'-CCCATTCGGTTTCCATTGTA-3' and reverse 5'-TCATCA GCCAACAGGAGGTT-3'; NF- $\mathrm{BB}$ (302 bp), forward 5'-TGC CTCCAGTGAGAAGAACA-3' and reverse 5'-GCACCAGAA GTCCAGGGTTA-3'; MCP-1 (302 bp), forward 5'-CAGCAG 
Table III. Effect of TS formula on hemodynamic indices $(n=8)$.

\begin{tabular}{|c|c|c|c|c|}
\hline Group & Blood viscosity & $\begin{array}{l}\text { Whole blood high shear } \\
\text { reductive viscosity }\end{array}$ & $\begin{array}{l}\text { Whole blood medium shears } \\
\text { reductive viscosity }\end{array}$ & $\begin{array}{l}\text { Whole blood low } \\
\text { shear reductive viscosity }\end{array}$ \\
\hline Control & $0.9 \pm 0.6$ & $3.33 \pm 1.06$ & $3.87 \pm 1.19$ & $6.95 \pm 1.93$ \\
\hline DN & $1.55 \pm 0.18^{\mathrm{a}}$ & $9.12 \pm 1.38^{\mathrm{a}}$ & $10.23 \pm 1.53^{\mathrm{a}}$ & $16.32 \pm 2.32^{\mathrm{a}}$ \\
\hline $\mathrm{TS}^{\text {Low }}$ & $1.17 \pm 0.22^{\mathrm{b}}$ & $5.7 \pm 2.21^{\mathrm{b}}$ & $6.99 \pm 2.82$ & $12.93 \pm 3.55^{\mathrm{b}}$ \\
\hline $\mathrm{TS}^{\text {Med }}$ & $1.05 \pm 0.08^{\mathrm{b}}$ & $6.12 \pm 0.59^{\mathrm{b}}$ & $8.24 \pm 5.52$ & $12.73 \pm 1.01^{\mathrm{b}}$ \\
\hline $\mathrm{TS}^{\mathrm{Hi}}$ & $1.03 \pm 0.08^{\mathrm{b}}$ & $5.17 \pm 1.45^{\mathrm{b}}$ & $5.93 \pm 1.62^{c}$ & $10.4 \pm 2.59^{\mathrm{b}}$ \\
\hline Valsartan & $1.15 \pm 0.22^{\mathrm{b}}$ & $5.34 \pm 1.32^{\mathrm{b}}$ & $6.42 \pm 1.52$ & $13.85 \pm 2.98^{\mathrm{b}}$ \\
\hline
\end{tabular}

${ }^{\mathrm{a}} \mathrm{P}<0.01$ vs. control group; ${ }^{\mathrm{b}} \mathrm{P}<0.01$ and ${ }^{\mathrm{c}} \mathrm{P}<0.05$ vs. DN group. Data are presented as the mean \pm standard error of the mean. TS, Tangshen; $\mathrm{TS}^{\text {Low }}$, low dose $(6.5 \mathrm{~g} / \mathrm{kg} /$ day $)$ of Tangshen formula; TS ${ }^{\text {Med }}$, medium dose $(13 \mathrm{~g} / \mathrm{kg} /$ day $)$ of Tangshen formula; TS ${ }^{\mathrm{Hi}}$, high dose of Tangshen formula ( $26 \mathrm{~g} / \mathrm{kg} /$ day) of Tangshen formula; Valsartan, $25 \mathrm{mg} / \mathrm{kg} /$ day of valsartan; DN, diabetic nephropathy.

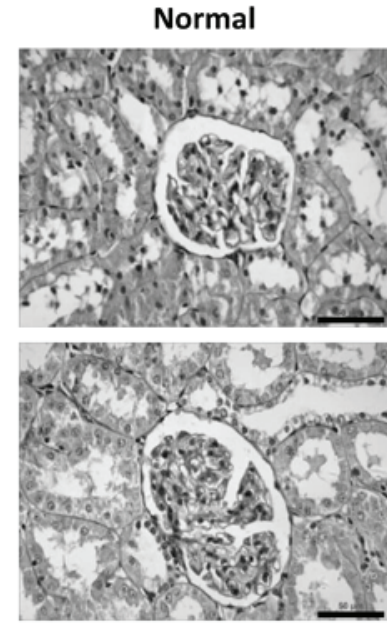

TSlow

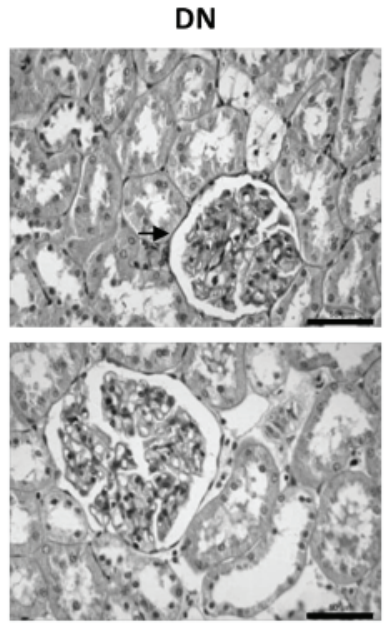

TS $^{\text {Med }}$

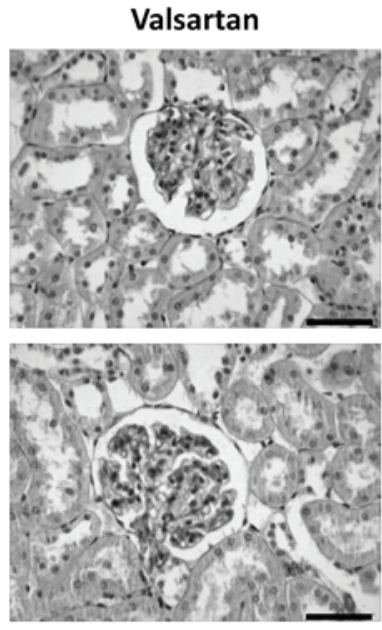

$\mathrm{TS}^{\mathrm{Hi}}$

Figure 1. Periodic Schiff reaction staining of the pathological changes in the kidneys of rats in the control group, DN group, TS ${ }^{\text {Low }}$ group, TS ${ }^{\text {Med }}$ group, $\mathrm{TS}^{\mathrm{Hi}}$ group and valsartan group. The arrow indicates the thickened basement membrane in the DN group. Scale bar, $50 \mu \mathrm{m}$. TS, Tangshen; TS ${ }^{\mathrm{Low}}$, low dose $(6.5 \mathrm{~g} / \mathrm{kg} / \mathrm{day})$ of Tangshen formula; TS ${ }^{\mathrm{Med}}$, medium dose (13 g/kg/day) of Tangshen formula; TS ${ }^{\mathrm{Hi}}$, high dose of Tangshen formula $(26 \mathrm{~g} / \mathrm{kg} / \mathrm{day})$ of Tangshen formula; Valsartan, $25 \mathrm{mg} / \mathrm{kg} /$ day of valsartan; DN, diabetic nephropathy.

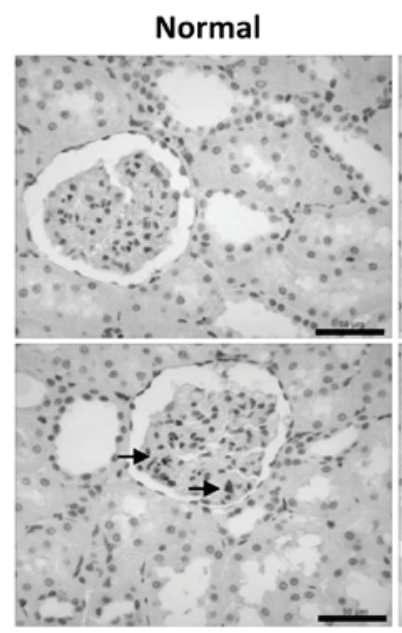

TS Low
DN

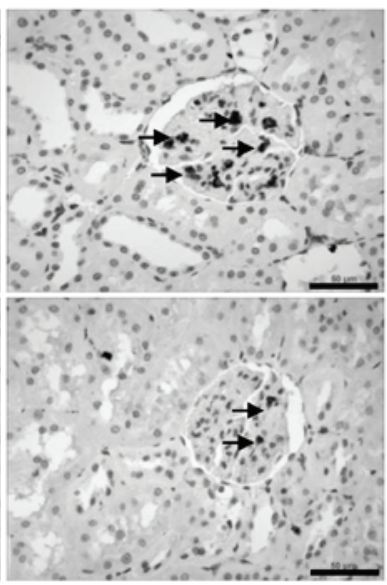

TS ${ }^{\text {Med }}$
Valsartan

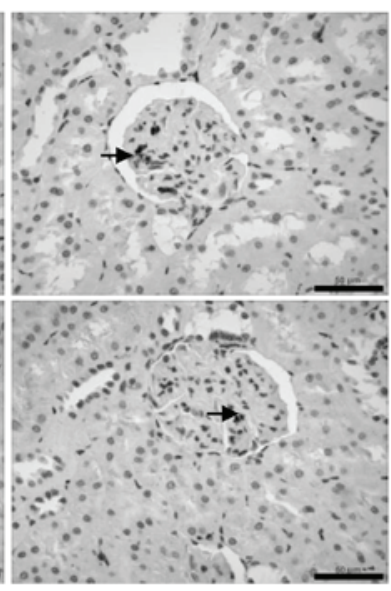

$\mathrm{TS}^{\mathrm{Hi}}$

Figure 2. Envision method immunohistochemical staining of the expression of microphages in the control group, DN group, TS ${ }^{\text {Low }}$ group, TS ${ }^{\text {Med }}$ group, TS ${ }^{\mathrm{Hi}}$ group and valsartan group. Arrows indicate the infiltrated macrophages in different group. Scale bar, $50 \mu \mathrm{m}$. TS, Tangshen; TS ${ }^{\text {Low }}$, low dose (6.5 g/kg/day) of Tangshen formula; TS ${ }^{\text {Med }}$, medium dose (13 g/kg/day) of Tangshen formula; $\mathrm{TS}^{\mathrm{Hi}}$, high dose of Tangshen formula (26 g/kg/day) of Tangshen formula; Valsartan, $25 \mathrm{mg} / \mathrm{kg} /$ day of valsartan; DN, diabetic nephropathy. 
Table IV. Microphage infiltration in different groups.

\begin{tabular}{lcc}
\hline Group & $\mathrm{N}$ & $\begin{array}{c}\text { Microphage infiltration } \\
\text { (count per field, }\end{array}$ \\
\hline Control & 8 & $0.93 \pm 0.28$ \\
DN & 8 & $8.80 \pm 1.16^{\mathrm{a}}$ \\
$\mathrm{TS}^{\text {Low }}$ & 8 & $5.47 \pm 0.79^{\mathrm{b}}$ \\
TS $^{\text {Med }}$ & 8 & $5.33 \pm 0.69^{\mathrm{b}}$ \\
TS $^{\text {Hi }}$ & 8 & $4.1 \pm 0.52^{\mathrm{b}}$ \\
Valsartan & 8 & $6.47 \pm 0.61^{\mathrm{c}}$ \\
\hline
\end{tabular}

${ }^{\mathrm{a}} \mathrm{P}<0.01$ vs. control group; ${ }^{\mathrm{b}} \mathrm{P}<0.01$ and ${ }^{\mathrm{c}} \mathrm{P}<0.05$ vs. DN group. Data are presented as the mean \pm standard error of the mean. TS, Tangshen formula; TS ${ }^{\text {Low }}$, low dose $(6.5 \mathrm{~g} / \mathrm{kg} /$ day $)$ of Tangshen formula; TS $^{\text {Med }}$, medium dose (13 g/kg/day) of Tangshen formula; $\mathrm{TS}^{\mathrm{Hi}}$, high dose of Tangshen formula ( $26 \mathrm{~g} / \mathrm{kg} /$ day) of Tangshen formula; Valsartan, $25 \mathrm{mg} / \mathrm{kg} /$ day of valsartan; DN, diabetic nephropathy.

GTGTCCCAAAGAAG-3' and reverse 5'-AGGCATCACATT CCAAATCAC-3'; MIF-1 (180 bp), forward 5'-CAGAACCGC AACTACAGCAA-3' and reverse 5'-CAGCAAGACTCGAAG AACAGC-3'; and GADPH (91 bp), forward 5'- GTGCTGAGT ATGTCGTGGAG-3' and reverse 5'-GTCTTCTGAGTGGCA GTGAT-3'. The RT-qPCR system contained: Sense primer $0.5 \mu \mathrm{l}(10 \mu \mathrm{M})$, antisense primer $0.5 \mu \mathrm{l}(10 \mu \mathrm{M})$, cDNA $(1 \mu \mathrm{l})$, double distilled $\mathrm{H}_{2} \mathrm{O}(8 \mu \mathrm{l})$ and SYBR Supermix from the iTaq $^{\mathrm{TM}}$ Universal Syber green One-Step kit $(10 \mu \mathrm{l})$. Reactions were incubated at $95^{\circ} \mathrm{C}$ for $2 \mathrm{~min}$ followed by $95^{\circ} \mathrm{C}$ for $10 \mathrm{sec}$, $55^{\circ} \mathrm{C}$ for $30 \mathrm{sec}$ and $72^{\circ} \mathrm{C}$ for $30 \mathrm{sec}$, for 40 cycles. Expression levels of mRNA were quantified by the $2^{-\Delta \Delta C q}$ method (8).

Western blot analysis. Samples were rinsed twice with cold PBS and then lysed on ice in radio immunoprecipitation assay lysis buffer and subsequently homogenized and centrifuged $\left(4^{\circ} \mathrm{C} ; 9,600 \mathrm{x} \mathrm{g}\right.$ for $\left.5 \mathrm{~min}\right)$ to collect the total protein. Protein content was determined using the BCA method. Supernatants were then transferred, mixed and boiled in sample buffer. Total protein (40 $\mu \mathrm{g} / \mathrm{lane})$ were separated by $12 \%$ SDS-PAGE and transferred to a polyvinylidene difluoride membrane. The membrane was incubated in blocking buffer consisting of 5\% fat-free milk dissolved in 1X Tris-buffered saline with Tween 20 (TBST; $10 \mathrm{mM}$ Tris-Base, $\mathrm{pH} 7.5,100 \mathrm{mM} \mathrm{NaCl}$ and $1 \%$ Tween 20) for $1 \mathrm{~h}$ at room temperature followed by incubation with monocyte chemoattractant protein-1 (MCP-1; sc-1785; 1:800), macrophage migration inhibitory factor (MIF; cat

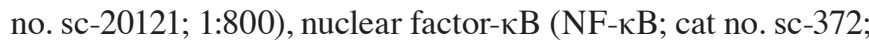
1:500) and sirtuin-1 (SIRT-1; cat no. sc-15404; 1:300) primary antibodies at $4^{\circ} \mathrm{C}$ overnight and $\beta$-actin (cat no. sc-58673; $1: 2,000)$ at room temperature for $1 \mathrm{~h}$. The membrane was subsequently washed with TBST and incubated at $37^{\circ} \mathrm{C}$ with IgG-horse radish peroxidase for $1 \mathrm{~h}$. The blot was visualized using an enhanced chemiluminescence reagent kit (GE Healthcare Life Sciences, Chalfont, UK) following four washes with TBS.

Statistical analysis. All data were presented as the mean \pm or + standard error of the mean. Differences between multiple groups were analyzed by one-way analysis of variance followed by Duncan's multiple range test. Differences between two groups were measured by Student's t-test, using SPSS 16.0 software (SPSS, Inc., Chicago, IL, USA). P $<0.05$ was considered to indicate a statistically significant difference.

\section{Results}

General conditions. Following STZ injection for 72 h, the condition observed in DN rats gradually declined as the rats exhibited dull fur, weight loss, were apathetic, unresponsive, had an increased appetite, thirst and frequency of urination and urine protein level, compared with the control group. Local ulceration, including foot ulcers, and deep skin ulcers were observed. Furthermore, the DN group displayed the most severe psychotic symptoms such as manic behavior. To some extent, groups with treatment exhibited less complications.

Change in body weight and metabolic parameters following treatment. As indicated in Table I, following modeling with STZ and high-fat glucose fodder, the body weight of rats significantly decreased when compared with the normal control $(\mathrm{P}<0.05)$. Moreover, TS formula improved this condition in a dose-dependent manner, which was similar to the therapeutic effect exhibited by valsartan treatment $(\mathrm{P}<0.05)$. Conversely, kidney weight and kidney hypertrophy index were significantly increased in the DN group compared with the control; however, this was improved in a dose-dependent manner in the groups, which were treated with different doses of TS formula $(\mathrm{P}<0.05$; Table I). Furthermore, these groups exhibited a similar weight and hypertrophy index as the valsartan treated group (Table I).

Fasting blood glucose (FBG), urinary albumin excretion rate (UAER), $\beta 2-M G$ and creatinine clearance rate $(\mathrm{Ccr})$ were evaluated following TS formula or valsartan treatment. The present results indicated that a high dose of TS formula significantly decreased FBG $(\mathrm{P}<0.01)$, UAER $(\mathrm{P}<0.05)$ and $\beta 2-\mathrm{MG}(\mathrm{P}<0.05)$ levels. Furthermore, a high dose of TS formula significantly improved Ccr compared with the DN group and the rate was similar to the Ccr exhibited in the valsartan group $(\mathrm{P}<0.05$; Table II). The whole blood low-, medium- and high-shear reductive viscosity and the blood viscosity were determined. The present results revealed that TS formula significantly reduced these hemorheology indices when compared with the DN group and a similar response was exhibited in the valsartan group $(\mathrm{P}<0.05$; Table III).

Renal pathological changes and macrophage infiltration. PAS staining indicated clear glomerular and renal tubular structures in the control group, with opened glomerular capillaries. Conversely, the glomerular volume was significantly increased $(\mathrm{P}=0.025)$ and the basement membrane was thickened in the DN group, with mesangial cell hyperplasia, expansion and renal tubule vacuolization observed. In the TS formula and valsartan treatment groups, these pathological changes were markedly decreased (Fig. 1). In addition, immunohistochemical results from all TS treatment groups indicated that TS formula significantly ameliorated macrophage infiltration that was exhibited in the DN group and the valsartan group (TS ${ }^{\text {Low }}$ vs. DN group, $\mathrm{P}=0.006$; TS $^{\text {Med }}$ 

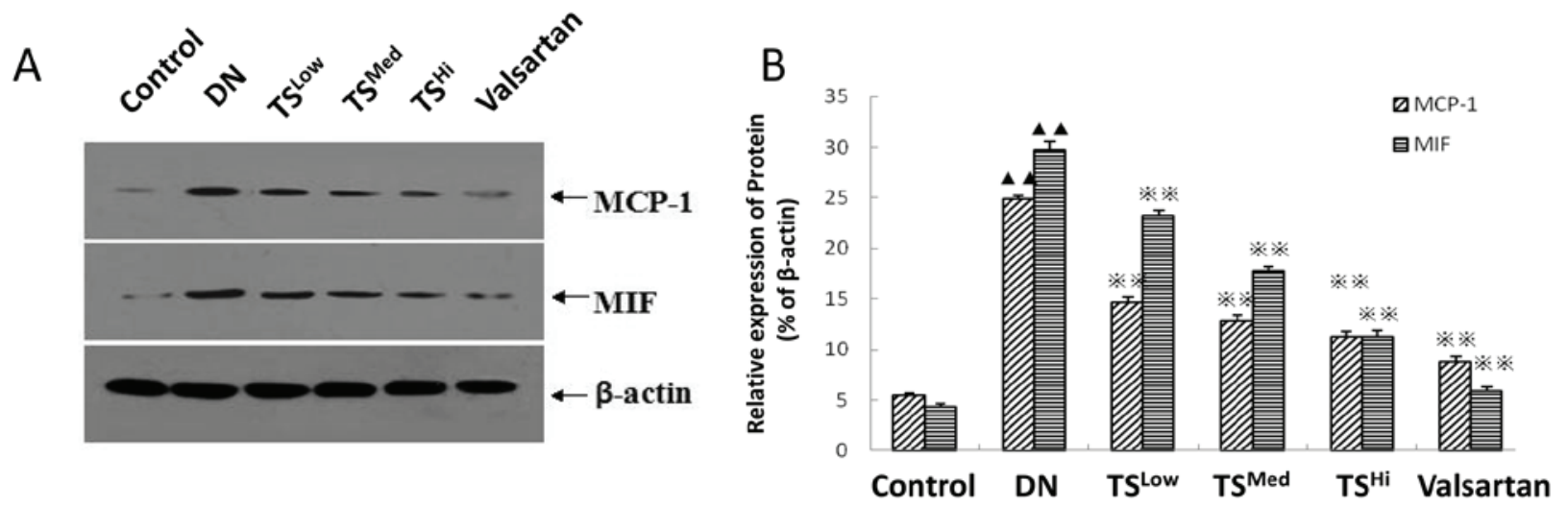

Figure 3. Protein expression levels of MCP-1 and MIF in the control group, DN group, $\mathrm{TS}^{\mathrm{Low}}$ group, $\mathrm{TS}^{\mathrm{Med}}$ group, $\mathrm{TS}^{\mathrm{Hi}}$ group and valsartan group were detected by (A) western blotting. (B) Relative protein expression levels of MCP-1 and MIF in all groups. Data are presented as the mean + standard error of the mean. ${ }^{\boldsymbol{\Delta}} \mathrm{P}<0.01$ vs. control, ${ }^{*} \mathrm{P}<0.01$ vs. DN group. TS, Tangshen; TS ${ }^{\text {Low }}$, low dose $(6.5 \mathrm{~g} / \mathrm{kg} / \mathrm{day})$ of Tangshen formula; $\mathrm{TS}^{\text {Med }}$, medium dose $(13 \mathrm{~g} / \mathrm{kg} / \mathrm{day})$ of Tangshen formula; $\mathrm{TS}^{\mathrm{Hi}}$, high dose of Tangshen formula ( $26 \mathrm{~g} / \mathrm{kg} / \mathrm{day}$ ) of Tangshen formula; Valsartan, $25 \mathrm{mg} / \mathrm{kg} / \mathrm{day}$ of valsartan; DN, diabetic nephropathy; MCP-1, monocyte chemoattractant protein-1; MIF, macrophage migration inhibitory factor.

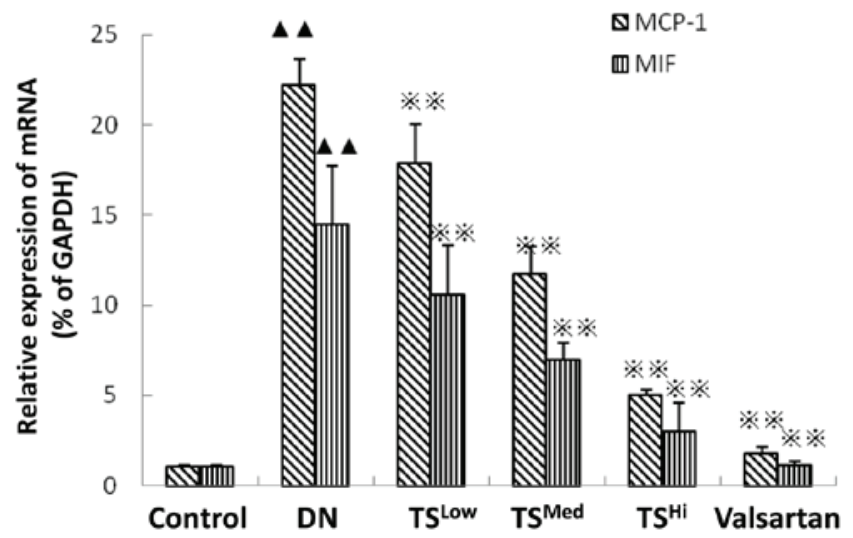

Figure 4. mRNA expression levels of MCP-1 and MIF in the control group, DN group, $\mathrm{TS}^{\mathrm{Low}}$ group, $\mathrm{TS}^{\mathrm{Med}}$ group, $\mathrm{TS}^{\mathrm{Hi}}$ group and valsartan group. Data are presented as the mean + standard error of the mean. ${ }^{\mathbf{\Lambda}} \mathrm{P}<0.01$ vs. control, ※* $\mathrm{P}<0.01$ vs. DN group. TS, Tangshen; TS ${ }^{\mathrm{Low}}$, low dose $(6.5 \mathrm{~g} / \mathrm{kg} / \mathrm{day})$ of Tangshen formula; TS $^{\text {Med }}$, medium dose $(13 \mathrm{~g} / \mathrm{kg} /$ day $)$ of Tangshen formula; $\mathrm{TS}^{\mathrm{Hi}}$, high dose of Tangshen formula (26 g/kg/day) of Tangshen formula; Valsartan, $25 \mathrm{mg} / \mathrm{kg} /$ day of valsartan; DN, diabetic nephropathy; MCP-1, monocyte chemoattractant protein-1; MIF, macrophage migration inhibitory factor.

vs. DN group, $\mathrm{P}=0.004$; $\mathrm{TS}^{\mathrm{Hi}}$ vs. $\mathrm{DN}$ group, $\mathrm{P}=0.003$; Fig. 2 , Table IV).

Effectiveness of TS formula on inflammatory proteins. RT-qPCR and western blotting results indicated that MIF and MCP-1 were significantly increased in the DN group compared with the normal control $(\mathrm{P}<0.01$; Figs. 3 and 4$)$. TS formula groups exhibited significantly decreased levels of these inflammatory factors, both at the protein level (Fig. 3) and mRNA level (Fig. 4), in a dose-dependent manner when compared with the DN group $(\mathrm{P}<0.01)$.

Furthermore, the SIRT1 and NF- $\kappa \mathrm{B}$ expression levels following different treatments were studied. Protein (Fig. 5) and mRNA (Fig. 6) expression levels of SIRT1 were significantly decreased and NF- $\kappa B$ expression levels were significantly increased in the DN group when compared with the normal control $(\mathrm{P}<0.01)$. Conversely, treatment with either low- medium- or high-dose TS formula reversed this trend, with the high-dose TS group exhibiting similar expression levels exhibited in the valsartan group.

\section{Discussion}

$\mathrm{DN}$ is the most common microvascular complication of diabetes and is one of the leading causes of end-stage renal disease worldwide (17). The primary risk factors contributing to the development and/or progression of DN include hyperglycemia, hypertension and dyslipidemia (18). The occurrence of DN is predominantly mediated by high glucose levels and microvascular hemodynamic changes and is further mediated by a variety of inflammatory cytokines in diabetic kidneys, which activate the inflammatory pathways (19). Capillary basement membrane thickening and mesangial matrix expansion of glomerular sclerosis precedes the final stages of DN (20). It has been suggested previously that the blood rheology changes are primarily responsible for microcirculation disorder exhibited in patients with diabetes (21). Inflammation has a vital role in the development of DN. High levels of blood glucose and hemodynamic disorder may cause apoptosis and shedding of kidney cells, including podocytes and proximal tubular epithelial cells, leading to the activation of leukocyte, along with the increasing recruitment of inflammatory chemokine factors (22).

MCP-1 is the strongest chemotactic factor for monocytes and urinary MCP-1 secretion levels are upregulated in multiple renal diseases, including DN $(23,24)$. MCP-1 has an important role in the pathogenesis of glomerular and progressive tubulointerstitial lesions via monocyte recruitment and activation (23). Increased urinary excretion of MCP-1 in patients with established DN (patients with micro- or macro albuminuria) is likely due to the enhanced production of MCP-1 in the renal tubules, which is presumably induced by excessive exposure to plasma protein filtered from the damaged glomeruli. However, MCP-1 may also upregulate the expression of CD11/18, which increases the adhesiveness of macrophages (25). Moreover, upregulated MCP-1 may lead to the activation of lysosomes and has a vital role in reactive oxygen species-induced kidney injury (20). 
A

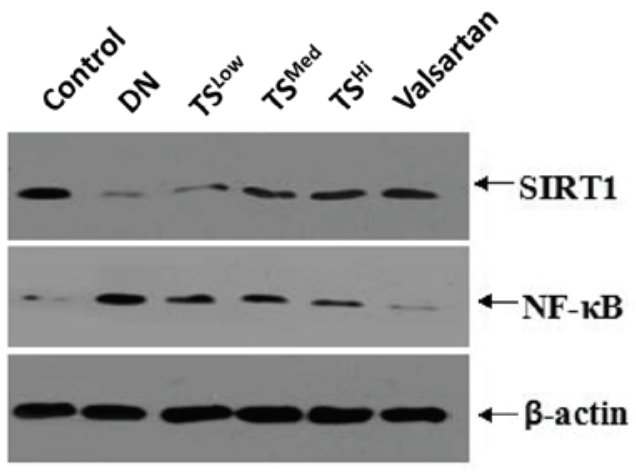

B

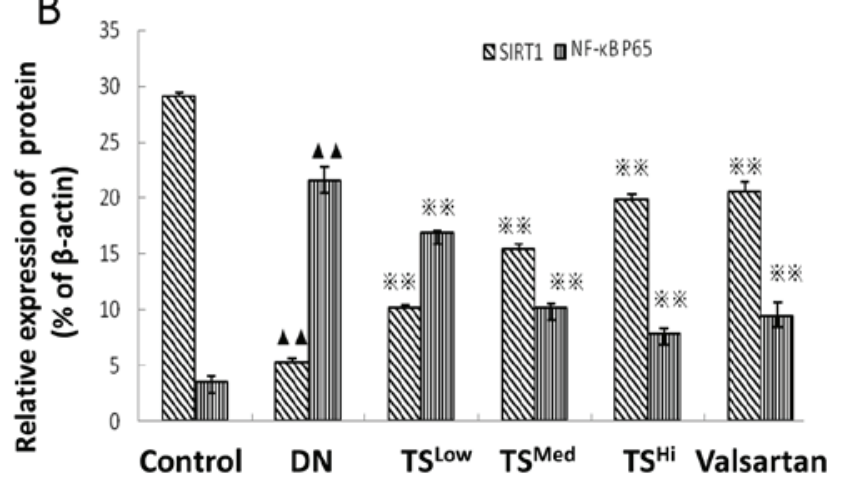

Figure 5. Protein expression levels of SIRT1 and NF-кB in the control group, DN group, $\mathrm{TS}^{\mathrm{Low}}$ group, $\mathrm{TS}^{\text {Med }}$ group, $\mathrm{TS}^{\mathrm{Hi}}$ group and valsartan group were detected by (A) western blotting. (B) Relative protein expression levels of SIRT1 and NF-kB in all groups. Data are presented as the mean + standard error of the mean. ${ }^{\boldsymbol{\Lambda}} \mathrm{P}<0.01$ vs. control, ${ }^{*} \mathrm{P}<0.01$ vs. DN group. TS, Tangshen; TS ${ }^{\text {Low }}$, low dose (6.5 g/kg/day) of Tangshen formula; $\mathrm{TS}^{\text {Med }}$, medium dose (13 g/kg/day) of Tangshen formula; $\mathrm{TS}^{\mathrm{Hi}}$, high dose of Tangshen formula ( $26 \mathrm{~g} / \mathrm{kg} /$ day) of Tangshen formula; Valsartan, $25 \mathrm{mg} / \mathrm{kg} /$ day of valsartan; DN, diabetic nephropathy;

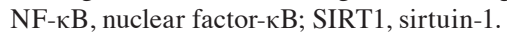
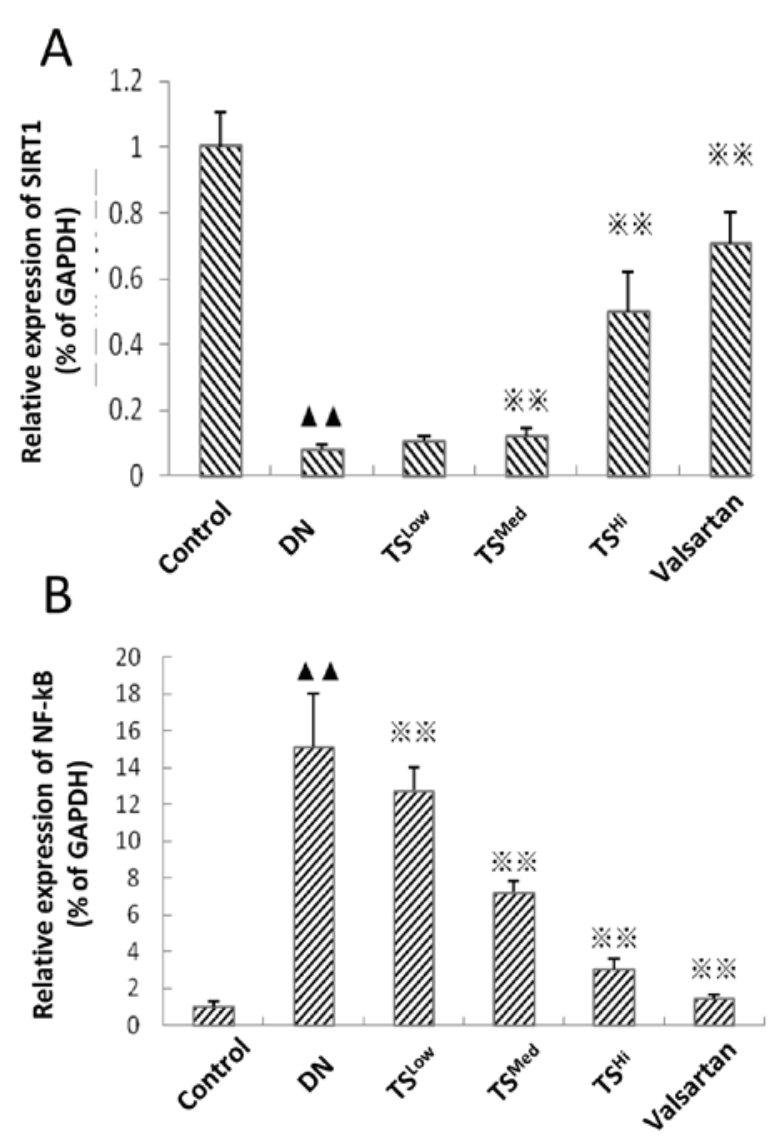

Figure 6. mRNA expression levels of (A) SIRT1 and (B) NF-kB in the control group, DN group, $\mathrm{TS}^{\mathrm{Low}}$ group, $\mathrm{TS}^{\mathrm{Med}}$ group, $\mathrm{TS}^{\mathrm{Hi}}$ group and valsartan group. Data are presented as the mean + standard error of the mean. ${ }^{\wedge} \mathrm{P}<0.01$ vs. control, ${ }^{*} \mathrm{P}<0.01$ vs. DN group. TS, Tangshen; TS ${ }^{\mathrm{Low}}$, low dose $(6.5 \mathrm{~g} / \mathrm{kg} /$ day $)$ of Tangshen formula; TS ${ }^{\text {Med }}$, medium dose $(13 \mathrm{~g} / \mathrm{kg} / \mathrm{day})$ of Tangshen formula; $\mathrm{TS}^{\mathrm{Hi}}$, high dose of Tangshen formula ( $\left.26 \mathrm{~g} / \mathrm{kg} / \mathrm{day}\right)$ of Tangshen formula; Valsartan, $25 \mathrm{mg} / \mathrm{kg} /$ day of valsartan; DN, diabetic

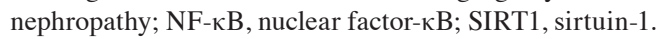

MIF has been described as a product of activated $\mathrm{T}$ cells that inhibits the migration of macrophages in vitro (26). Low levels of glucocorticoids upregulate MIF expression via a counter regulatory mechanism whereby MIF can override the glucocorticoid effects, which suggests the presence of this component has a vital role in amplifying pro-inflammatory loop responses (27). Furthermore, Bruchfeld et al revealed that MIF are highly expressed in chronic nephrosis (28).

Previous results have indicated that SIRTs may be identified and activated in the kidneys, liver, spleen, lung, heart, muscle, brain, testis, ovaries, thymus, pancreas and white or brown adipose tissue $(29,30)$. Localization of SIRT proteins differs in cells and may develop various physiologic and pathologic metabolic effects under certain stress conditions (29). SIRT1 is the most studied member of the sirtuins, likely due to its generalized effects on the cell cycle, mitochondrial metabolism, energy homeostasis, inflammation, oxidative stress and apoptosis (30). SIRT1 may have a key role in the regulation of whole body metabolic homeostasis, and downregulation of SIRT1 in visceral adipose tissue may contribute to the metabolic abnormalities that are associated with visceral obesity in diabetic and obese women (31). SIRT1-deficient mice exhibit low levels of serum glucose and insulin (32). Molecular evidence has indicated that expression of SIRT1 is able to decrease the acetylation of NF- $\mathrm{kB}$ and reduce the toxic effect of cisplatin on kidney tubules. Additionally, a previous study indicated that abnormal activation of NF- $\mathrm{KB}$ may promote inflammatory responses and autoimmune responses, which results in the expansion of the extracellular matrix, inflammatory cell infiltration and renal tubule interstitial fibrosis (33).

Through long-term clinical practice, traditional Chinese medicine proposed a theory where kidney damage is caused by turbidity toxin called the 'damage of kidney by poison' theory. It is believed that phlegm stagnation implicates the renal microcirculation system, qi stagnation, stasis and pathogenic factor accumulation into turbidity toxins and that the toxins damage the renal microcirculation system (4). Damage to the renal microcirculation system is considered the most important pathogenesis factor of DN, which is a condition associated with the production of various inflammatory factors, including MCP-1 and NF- $\mathrm{KB}$ (34). The essence of stasis refers to thrombosis or embolism complications in the kidney, which is the typical pathology of DN in traditional Chinese medicine and its symptoms include a change in renal hemodynamics and involves mesangial cell proliferation 
and extracellular matrix accumulation (34). This may lead to the onset of glomerulosclerosis in patients with DN (34). The accumulation of turbidity toxin is usually caused by blood stasis, phlegm and moisture (35). The mechanism of DN inflammatory pathogenesis proposed by modern western medicine provides theoretical circumstantial evidence for the pathological framework for the 'damage of kidney by poison' theory (35).

Western and traditional Chinese medicine researchers agree that the changes in hemodynamics and inflammatory factors are important pathological changes during the development of DN (35). TS formula is produced from six types of traditional Chinese medicine, namely Astragalus, Radix puerariae, Lucid ganoderma, Ligustrum lucidum, Rheum officinale and Salvia miltiorrhiza. Astragalus and Radix puerariae (15). Astragalus is beneficial for supplementing Qi and strengthening the immune system (36). Radix puerariae is used as an antipyretic and promotes the secretion of saliva (37). The combination of these two agents is beneficial in supplementing Qi and promoting the secretion of saliva (37). Lucid ganoderma could supplement Qi of the liver, heart, spleen, lungs and kidneys (37). Ligustrum lucidum fruit supplements kidney fluids, nourishes Yin of the liver and may reduce inflammation. In traditional Chinese medicine, Rhubarb cooked with rice wine is beneficial for promoting circulation, removing stasis, and when combined with Salvia miltiorrhiza, it may promote circulation and clear the body of phlegm senescence and blood stasis (37).

In the present study, following the successful establishment of the DN model, the effectiveness of TS formula was investigated. The present results revealed that TS formula significantly improved the physical condition of rats with DN and ameliorated kidney hypertrophy indices in a dose-dependent manner, which was similar to the effect exhibited with valsartan treatment. Furthermore, TS formula significantly decreased the pathological changes, including FBG, UAER, $32-\mathrm{MG}$, hemorheology index, and mesangial cell hyperplasia and expansion. These results indicated that TS formula could effectively improve the pathological state of patients with DN and protect the kidneys.

In the present study, western blotting and RT-qPCR results demonstrated that the protein and mRNA expression levels of inflammatory factors, MIF and MCP-1, were significantly decreased following treatment with TS formula, which indicated that TS formula may have an effective role in preventing inflammatory responses. The anti-inflammatory effect may be closely associated with the upregulation of SIRT1 and the ensuing downregulation of $\mathrm{NF}-\kappa \mathrm{B}$ that was exhibited in the present results, in order to block the key regulative protein in the inflammatory responses and protect the kidney.

In conclusion, TS formula elicited a prominent therapeutic effect by targeting two key pathological changes in symptoms: The changes in hemodynamics of DN; and the infiltration of inflammatory factors, which may be closely related to its pharmacological action on activating SIRT1 expression and $\mathrm{NF}-\kappa \mathrm{B}$ downregulation, leading to a final inhibitory effect of pro-inflammatory factors. However, the necessary constituents of TS formula require further study and the functions of each constituent in the pathology of DN requires further elucidation.

\section{Acknowledgements}

The present work was supported by the National Natural Science Fund (grant no. 81273615) and Zhejiang Provincial Natural Science Foundation of China (grant no.LY16H270008).

\section{References}

1. Martynyuk L, Martynyuk L, Ruzhitska $\mathrm{O}$ and Martynyuk O: Effect of the herbal combination Canephron $\mathrm{N}$ on diabetic nephropathy in patients with diabetes mellitus: Results of a comparative cohort study. J Altern Complement Med 20, 472-478, 2014.

2. Pan XR, Yang WY, Li GW and Liu J: Prevalence of diabetes and its risk factors in China, 1994. National Diabetes Prevention and Control Cooperative Group. Diabetes Care 20: 1664-1669, 1997.

3. Vikram A, Tripathi DN, Kumar A and Singh S: Oxidative stress and inflammation in diabetic complications. Int J Endocrinol 2014: 679754, 2014.

4. Guo J, Chen H, Song J, Wang J, Zhao L and Tong X: Syndrome differentiation of diabetes by the traditional chinese medicine according to evidence-based medicine and expert consensus opinion. Evid Based Complement Alternat Med 2014: 492193, 2014

5. Tong XL, Dong L, Chen L and Zhen Z: Treatment of diabetes using traditional Chinese medicine: Past, present and future. Am J Chin Med 40: 877-886, 2012.

6. Bensky D and Gamble A: Herbs that drain dampness. Chin Her Med Mat Med 3: 265-266 (In Chinese).

7. Wang J, Wang Q, Li L, Li Y, Zhang H, Zheng L, Yang L, Zheng Y, Yang Y, Peng G, et al: Phlegm-dampness constitution: Genomics, susceptibility, adjustment and treatment with traditional Chinese medicine. Am J Chin Med 41: 253-262, 2013.

8. Edward FBIV: TCM Dampness and Food Therapy. Acupunct Today 09: 2008.

9. Wang Z, Wang J and Chan P: Treating type 2 diabetes mellitus with traditional chinese and Indian medicinal herbs. Evid Based Complement Alternat Med 2013: 343594, 2013.

10. Zhou J, Gao C and Wu ST: Pathogenic Mechanism of toxic cloud. J TCM Univ Hunan 29: 22-27, 2009 (In Chinese).

11. Gohda T: Treatment of diabetic nephropathy, particularly focusing on drug therapy. Nihon Rinsho 74 (Suppl 2): S202-S206, 2016.

12. Saxena U, Timmer RT and Pillarisetti S: New approaches for treatment of diabetic nephropathy: The endothelium as a target for drug discovery. Expert Opin Ther Targets 5: 539-545, 2001.

13. Sang Y, Wang XB and Han Q: Effects of tangshenkang capsule on diabetic nephropathy. Zhongguo Zhong Xi Yi Jie He Za Zhi 16: 398-401, 1996 (In Chinese).

14. Xu XY and Ye SW: Efficacy assessment of treating post-stroke shoulder-hand syndrome patients of yin deficiency yang hyperactivity with blood stasis stagnation collaterals syndrome by yishen tongluo decoction. Zhongguo Zhong Xi Yi Jie He Za Zhi 34: 1069-1073, 2014 (In Chinese).

15. Zhao T, Sun S, Zhang H, Huang X, Yan M, Dong X, Wen Y, Wang H, Lan HY and Li P: Therapeutic effects of tangshen formula on diabetic nephropathy in rats. PLoS One 11: e0147693, 2016.

16. Yang X, Zhang B, Lu X, Yan M, Wen Y, Zhao T and Li P: Effects of tangshen formula on urinary and plasma liver-type fatty acid binding protein levels in patients with type 2 diabetic kidney disease: Post-hoc findings from a multi-center, randomized, double-blind, placebo-controlled trial investigating the efficacy and safety of tangshen formula in patients with type 2 diabetic kidney disease. BMC Complement Altern Med 16: 246, 2016.

17. Lim AKh: Diabetic nephropathy - complications and treatment. Int J Nephrol Renovasc Dis 7: 361-381, 2014.

18. Meier M and Haller H: Diabetic nephropathy - current concepts in early diagnosis and treatment of diabetic microvascular complications. Herz 29: 496-503, 2004 (In German).

19. Donate-Correa J, Martín-Núñez E, Muros-de-Fuentes M, Mora-Fernández C and Navarro-González JF: Inflammatory cytokines in diabetic nephropathy. J Diabetes Res 2015: 948417, 2015.

20. Fakhruddin S, Alanazi W and Jackson KE: Diabetes-induced reactive oxygen species: Mechanism of their generation and role in renal injury. J Diabetes Res 2017: 8379327, 2017.

21. Cho YI, Mooney MP and Cho DJ: Hemorheological disorders in diabetes mellitus. J Diabetes Sci Technol 2: 1130-1138, 2008. 
22. Gajjala PR, Sanati M and Jankowski J: Cellular and molecular mechanisms of chronic kidney disease with diabetes mellitus and cardiovascular diseases as its comorbidities. Front Immunol 6: $340,2015$.

23. Yi B, Hu X, Zhang H, Huang J, Liu J, Hu J, Li W and Huang L: Nuclear NF- $\kappa \mathrm{B}$ p65 in peripheral blood mononuclear cells correlates with urinary MCP-1, RANTES and the severity of type 2 diabetic nephropathy. PLoS One 9: e99633, 2014.

24. Xu ZZ, Wang M, Wang YJ, Niu HX, Li XY, Zhou WD, Zhu Y and Long HB: Effect of nitrotyrosine on renal expressions of NF- $\kappa \mathrm{B}, \mathrm{MCP}-1$ and TGF- $\beta 1$ in rats with diabetic nephropathy. Nan Fang Yi Ke Da Xue Xue Bao 33: 346-350, 2013 (In Chinese).

25. Aziz MH, Cui K, Das M, Brown KE, Ardell CL, Febbraio M, Pluskota E, Han J, Wu H, Ballantyne CM, et al: The upregulation of integrin $\alpha \mathrm{D} \beta 2(\mathrm{CD} 11 \mathrm{~d} / \mathrm{CD} 18)$ on inflammatory macrophages promotes macrophage retention in vascular lesions and development of atherosclerosis. J Immunol 198: 4855-4867, 2017.

26. Toso C, Serre-Beinier V, Emamaullee J, Merani S, Armanet M, Wojtusciszyn A, Bosco D, Calandra T, Roger T, Morel P, et al: The role of macrophage migration inhibitory factor in mouse islet transplantation. Transplantation 86: 1361-1369, 2008.

27. Lan HY, Yang N, Metz C, Mu W, Song Q, Nikolic-Paterson DJ, Bacher M, Bucala R and Atkins RC: TNF-alpha up-regulates renal MIF expression in rat crescentic glomerulonephritis. Mol Med 3: 136-144, 1997.

28. Bruchfeld A, Carrero JJ, Qureshi AR, Lindholm B, Barany P, Heimburger O, Hu M, Lin X, Stenvinkel P and Miller EJ: Elevated serum macrophage migration inhibitory factor (MIF) concentrations in chronic kidney disease (CKD) are associated with markers of oxidative stress and endothelial activation. Mol Med 15: 70-75, 2009.
29. Morrissey J and Klahr S: Transcription factor NF-kappaB regulation of renal fibrosis during ureteral obstruction. Semin Nephrol 18: 603-611, 1998.

30. Pan Y, Zhang X, Wang Y, Cai L, Ren L, Tang L, Wang J, Zhao Y, Wang Y, Liu Q, et al: Targeting JNK by a new curcumin analog to inhibit NF-kB-mediated expression of cell adhesion molecules attenuates renal macrophage infiltration and injury in diabetic mice. PLoS One 8: e79084, 2013.

31. Bible E: Diabetic nephropathy: Sirt1 attenuates diabetic albuminuria. Nat Rev Nephrol 9: 696, 2013.

32. Kitada M and Koya D: SIRT1 in type 2 diabetes: Mechanisms and therapeutic potential. Diabetes Metab J 37: 315-325, 2013.

33. Sakao Y, Kato A, Tsuji T, Yasuda H, Togawa A, Fujigaki Y, Kahyo T, Setou M and Hishida A: Cisplatin induces Sirt1 in association with histone deacetylation and increased Werner syndrome protein in the kidney. Clin Exp Nephrol 15: 363-372, 2011.

34. Imig JD and Ryan MJ: Immune and inflammatory role in renal disease. Compr Physiol 3: 957-976, 2013.

35. Xie W and Du L: Diabetes is an inflammatory disease: Evidence from traditional Chinese medicines. Diabetes Obes Metab 13: 289-301, 2011.

36. Yang XJ and Xiao SY: Regularity of drug use of patents in force of traditional Chinese medicine compounds in preventing and treating cardiovascular diseases on basis of frequency analysis. Zhongguo Zhong Yao Za Zhi 37: 2661-2666, 2012 (In Chinese).

37. Su SY and Hsieh CL: Anti-inflammatory effects of Chinese medicinal herbs on cerebral ischemia. Chin Med 6: 26, 2011. 